\title{
The Self in an Interpretive Community
}

\begin{abstract}
In this chapter we continue the exploration of ethics as embedded in thought, emotion, and action. We begin by discussing two cases, one of which presents a sharp conflict, one of which does not, and both of which use the goal-directed, concrete language of clinical medicine as the health professionals work out their differences, the challenges presented by the cases, and ultimately what to do. We then introduce the notion of interpretive community as a way of understanding how ethics is embedded in the very framework of health care.
\end{abstract}

Keywords Health care - Clinical ethics - Implicit ethical framework Informal ethical discourse - The self - Interpretive community Morality of ordinary practice - Strategic flexibility - Collaborative problem solving $\cdot$ Hidden curriculum

\section{Two VignetTes}

\section{Strategic Flexibility}

In a case conference centered on a deteriorating, 77-year-old, terminally ill woman with colon cancer, the medical residents were in sharp conflict with the patient's gastroenterologist, who wanted to conduct further tests to determine exactly how far the cancer had progressed. The residents

(C) The Author(s) 2018

S. Scher and K. Kozlowska, Rethinking Health Care Ethics, https://doi.org/10.1007/978-981-13-0830-7_7 
believed that the tests were unnecessarily invasive, however, and pressed the GI specialist to explain why the tests were necessary. That is, given that the tests were invasive and would cause serious discomfort to the patient, what would they achieve? How could they be justified? The GI specialist admitted that the test results would not change the course of treatment, but he was also adamant about the importance-to him-of determining the patient's exact medical condition before he provided any further treatment. It was a complete standoff. Toward the end of the hour scheduled for the case conference, however, the GI specialist used the phrase "not while I'm her doctor." This phrase suggested a way out of this sharp, unyielding confrontation: would the doctor consider transferring the patient to one of the hospital's other gastroenterologists? He had no difficulty agreeing - an outcome that respected both his concerns and professional values and those of the residents. The transfer of care to another specialist, when explained to the family, was also one that they found acceptable.

Perhaps the most striking thing about this case is the openness and directness of the confrontation and discussion. The residents assertively express their views, and the GI specialist pushes back just as hard. Neither side is prepared to give an inch. It is fair to say that what we have here is a collision of professional selves - and of what we described in the previous chapter as their patterns of strategic flexibility. As deep expressions of their professional selves, both sides resist what the other is demanding.

Also noteworthy about this case is the complete absence of ethical terminology. The entire discussion took place without any mention of rights, principles, obligations, or any such concepts. The residents spoke of unnecessary, intrusive care, and the GI specialist spoke only of what he was prepared to do, or not, as the woman's physician. And as a senior physician with established patterns of practice, he did not and would not proceed with any treatment decision without having an evidence-based understanding of the patient's medical condition. This position was an expression of his capacity for strategic flexibility - that is, his willingness to extend himself, or not, in relation to the expectations or demands of others - that had long been established as part of his fast thinking ("I don't work that way") (see Chapter 6). It was part of the framework within which he thought about patient care. But it was not experienced, by him, as an "ethical" dimension of medical care. It was, for him, simply good medicine. Likewise, what the residents were demanding was, 
for them, simply good medicine. And it was only by thinking "outside the box" that the two sides were able to reach a mutually agreeable accommodation.

Finally, if one interprets this situation as involving each side's conception of their ethical obligations, one is missing the two central implications of the case: first, ethics is embedded, rather than explicit, in the case (and, more generally, in the practice of health care); and second, ethics, as here, is largely experienced in terms of the concrete, goal-oriented clinical decisions that health professionals make, often in collaboration with patients and families.

\section{Collaborative Problem Solving}

Oscar, a 16-year-old boy who lived with his parents in a country town, was referred to a tertiary care hospital for assessment of narcolepsy after a year and a half of sleeping seventeen hours a day following a flu-like illness. After confirming the diagnosis (via a sleep study), the intervention in the adolescent medicine ward included scheduled naps for the narcolepsy, school attendance at the hospital school, physiotherapy (to address a deconditioned physical state), and a trial of medication (modafinil) for narcolepsy. Oscar's presentation symptoms largely improved, but while awake he began to experience foggy thinking, vagueness, and memory problems (e.g., not recognizing where he was or remembering what had occurred that day), punctuated by what appeared to be dissociative episodes, in which he spoke in a baby voice and acted like an out-of-control toddler. Because the etiology of these new symptoms was unknown, a neurologist and a consultation-liaison psychiatrist were brought in. Potential explanations included a neuro-inflammatory process, a functional stress-related disorder (Oscar had a long history of anxiety), or an unusual feature of the narcolepsy.

The pattern of Oscar's dissociative episodes was unknown, as were the possible triggers. It was also unknown whether the mother's constant attention to Oscar's symptoms was itself triggering or exacerbating his problems. A complicating factor was that much the observational data about Oscar was coming from the mother herself because of the comparatively light nursing staff on the medical ward. The psychiatrist suggested that all of these concerns could be addressed through a two-week admission to the mental health ward, a small ward with ongoing, 24-hour nursing observation and further opportunities for psychometric assessment.

This suggestion met with various reasoned objections: 
1. The nurses on the mental health ward perceived his current problems as primarily medical and neurological, with the consequence that they saw the transfer as inappropriate and also as creating unnecessary work.

2. Oscar himself had lost his patience with being hospitalized, and wanted to go home. He also had no recollection of his dissociative episodes, so one of the main reasons for his hospitalization was not even within his conscious memory.

3. In contrast to the arrangement on the medical ward, Oscar's mother would not be able to sleep by Oscar's bed. The mother was worried that he would, upon awaking, not know where he was (because of his memory problems) and also that the nursing staff would be unable to administer his narcolepsy medication (of necessity, before he was fully awake, using a technique that the mother herself had developed).

4. Hospital administration was pushing for discharge since the admission, from their perspective, was already overly long.

The treating team (sleep physician, adolescent medicine physician, neurologist, and psychiatrist, plus adolescent medicine, neurology, and psychological medicine residents) understood the situation as involving a set of interrelated problems, each of which needed to be addressed in its own terms.

1. After the team explained to the mental health nurses that the memory problems and dissociative episodes were likely stress related and that the nurses' observations and psychometric assessment were critical in understanding Oscar's problems, the nurses understood why the transfer was needed.

2. The mother, at the team's suggestion, explained to Oscar that, at home, she would be unable to manage his dissociative episodes.

3. The treating team, nurses, and mother reached an agreement that for the first three days of the transfer, she would arrive at Oscar's beside prior to his waking, administer his medication, and train the nurses how to do the same.

4. The team explained to administration that unless Oscar's problems were brought under control now, he would simply return later, and in worse condition than at present.

During the resulting admission, nursing observations revealed that Oscar's memory problems and dissociative events typically occurred when Oscar's nighttime sleep had been unsettled (typically involving a range of violent movements). A presumptive disorder of REM sleep was identified 
and medicated. With improved sleep, the daytime symptoms improved dramatically, allowing for Oscar's discharge and return to school.

This case is noteworthy for what might have gone wrong but did not. Early on, the treatment team's relationship with the mother could well have deteriorated and, on the spot, ended the intervention. She might, in particular, have resented the treatment team's suggestions that independent reports of Oscar's functioning were important to obtain and that she, because of her high level of concern for her son and her attention to his symptoms, ${ }^{1}$ may by her very presence have been exacerbating or even triggering Oscar's symptoms. She may also have refused her son's transfer to the mental health ward, given that she would no longer be able to stay overnight in his room. But the staff handled the delicate matter of her reports gently and directly, and they were able to work out a compromise concerning her overnight stays, one that addressed her main concerns but that also enabled the team to assess (beginning three days after the transfer) the mother's potential contribution to her son's medical situation. The initial opposition of the mental health ward nurses and the hospital administration was also handled directly and with aplomb. Oscar could himself have simply refused to continue with the hospitalization, but the mother, fully informed of the medical situation, was able to explain why a return home was not possible from her perspective without a further stay in the hospital. Finally, almost invisible in the case is the seamless cooperation of an increasing number of specialists as the case developed. Absent is any suggestion of turf or professional prerogatives; throughout the case, the treatment team, ever expanding, operates as a unified whole. Likewise, since the case was so well managed and the communication so effective, none of the care providers felt that their own goals or standards had been compromised in any way; their professional selves were fully expressed.

Also noteworthy about this case, as with the first (concerning strategic flexibility), is that it is completely lacking in ethical terminology. The entire case occurs within the goal-directed framework of providing good medical care to this particular patient. ${ }^{2}$ The mother asserts no rights; the patient asserts no rights; the mental health nurses assert no rights; and the various members of the treatment team assert no rights. No ethical principles are invoked. Good, respectful, effective care is 
provided, with the potential conflicts addressed only by reference to what needed to be done to help the patient.

A final element of this case is the implicit teaching-whether one calls it the informal or hidden curriculum of medical training-of the adolescent medicine, neurology, and psychological medicine residents. What they witness and, indeed, participate in is the direct, effective, humanly responsive management of a medically difficult situation, again without any mention of ethical concepts as such. The treating team aimed to provide good medical care, and they did.

To take a step back, it is important to note that these cases are not unlike many of those that come before hospital ethics committees, and that some bioethicists conceive of their work along the above linesas aiding health professionals, patients, and families to reach mutually agreeable outcomes. What we are adding, in this book, is an overall framework within which to understand such efforts.

\section{INTERPRETIVE COMMUNITIES}

The two cases above bring to mind what the anthropologist Arthur Kleinman observed about the moral experience of health professionals. As mentioned in our introductory chapter, Kleinman (1999, pp. 71-72) sees their experience, like that of all other persons, as embedded in "the local processes (collective, interpersonal, subjective) that realize (enact) values in ordinary living" and that connect "affect and cognition with cultural meanings, moral norms and collective identity with sense of self," with the consequence that "moral experience and personal experience are interfused, value with emotion." That's just what we see in our two cases. The ethics-what we have called informal ethical discourseis embedded in the social environment as what people do, what people feel, and what people think.

In this context it is helpful to borrow a notion at the interface of sociology and literary criticism. In his 1980 book Is There a Text in This Class? and as subsequently elaborated (1989) in Doing What Comes Naturally, the literary theorist Stanley Fish uses the expression interpretive community to describe "not so much a group of individuals who shared a point of view, but a point of view or way of organizing experience" that itself defines and includes the practices and standards that enable individuals to act together, or to be "constituted," as members of a particular community $(1989$, p. 141). Though each member of a community thinks and acts 
individually, the "interpretive acts" are not themselves individual but communal. They take place within the "understood practices and assumptions" of an institution $(1980$, p. 306). In that respect each member of a particular interpretive community is what Fish calls an "embedded practitioner whose standards of judgment, canons of evidence, or normative measures are extensions" of the community itself (1989, p. 144).

The role of practices and standards, along with their interconnections, is crucial in understanding what interpretative communities are and how they function over time. Interpretive communities, for Fish, can be understood as sets of institutional practices with "assumed distinctions, categories of understanding, and stipulations of relevance and irrelevance" (1989, p. 141). With this orientation toward shared processes and standards rather than toward substantive agreement and closure, interpretive communities are not monolithic but allow both for a diversity of viewpoints and, within the bounds of a community's practices, for robust individual expression. Indeed, it is this robust (and diverse) individual expression, coupled with the ever-evolving social context in which community activity (= interpretation) occurs, that enables an interpretive community to change and adapt over time.

Examples of interpretive communities are everywhere, and we are all familiar with them. Fish's immediate interest was literary theory and the interpretive community of literary critics. What he said about literary criticism would apply just as well, and in the same terms, to any academic field such as economics or history or philosophy or any of the sciences. Fish himself also gave some careful, probing attention to the law, especially the judiciary, and other writers have applied the notion of interpretive community to journalism and to international law, among other fields. What distinguishes these different interpretive communities are their distinctive, interconnected sets of practices - their shared goals, standards, processes, positions, and social roles, all within an evolving social context. Disagreement within interpretive communities is common but is managed (and built upon, generating change and progress) through the shared understandings that constitute each separate community. Disagreement between different interpretive communities is also common, but how it is managed is an open question; there are not necessarily any shared understandings and processes to manage conflict or even discussion. Disagreements can be gentle or sharp, settled or not. By the same token, social, political, or legal forces can impinge, intentionally or not, on any particular interpretive community. But the actual 
impact of that impingement will depend upon the way in which the community incorporates it, or not, into its existing practices. External forces will, that is, inevitably be reinterpreted as they meet the existing practices of an ongoing interpretive community.

\section{Health Care as Overlapping Interpretive Communities}

Given the above characterization of interpretive communities, our suggestion is that each field of health care-for example, medicine, nursing, social work, or clinical psychology_can be understood as an interpretive community, and that the overarching field of health care can be understood as comprising overlapping interpretive communities. We suspect, in particular, that health professionals will see themselves in the following quote from Fish's Doing What Comes Naturally, which concisely describes what it is to be a member of an interpretive community and which also, in effect, summarizes the entire analysis set forth in the preceding section:

To think within a practice is to have one's very perception and sense of possible and appropriate action issue "naturally"-without further reflection-from one's position as a deeply situated agent. Someone who looks with practice-informed eyes sees a field already organized in terms of perspicuous obligations, self-evidently authorized procedures, and obviously relevant pieces of evidence. (pp. 386-387)

It is this naturalness of perception, action, and reasoning that is so apparent in the two cases that opened this chapter. It is a naturalness, too, that describes the experience of professionals working within particular fields of health care, where one simply is, and works as, a doctor or nurse or social worker or clinical psychologist. A health professional doesn't step back and say, for example (except perhaps in very unusual circumstances), "Since I am a nurse, this is how I am supposed to think." Instead, a trained nurse simply (or "naturally") has come to perceive, think, and act as a nurse or, perhaps even better, as the particular nurse that, through training and experience, he or she has become.

It is helpful to note that this naturalness of self-expression- "without further reflection"-directly parallels our discussion of fast thinking in Chapter 6. Much of the thinking that occurs naturally and without reflection would be characterized as fast thinking and as elements of a 
person's expertise, or working knowledge, in a particular field of health care. Drawing on Fish's quotation above, these elements include perception, range of options, actions, obligations, procedures, and rules of evidence/relevance. Depending upon the situation, however, fast thinking can give way to slow thinking, as when the standard way of thinking about a problem doesn't quite fit a particular case, or when disagreement or conflict arises and the situation needs to be sorted out through explicit discussion, or when a person is pushed to extend the limits of his or her strategic flexibility. And even when disagreement is sharp, it typically takes place against a rich background of shared working knowledge (characterized broadly, as above).

In the care of any particular patient or group of patients, or in formulating policies of one kind or another, professionals from different fields of health care regularly interact, bringing with them each field's distinctive interpretive framework. Much of what is in these different frameworks actually overlaps since central to all of them is the quest to provide health care to patients with some form of science- or evidence-based interventions. Nevertheless, nurses might see a particular case or policy one way, social workers another, clinical psychologists another, and doctors another. For any sort of consensus to be reached, these differences need to be worked out. A baseline for reaching consensus is each field's pattern of strategic flexibility, which defines the range of solutions tolerable to the professionals in each field. Negotiation and discussion can then take place within those boundaries, though with the understanding that those boundaries might themselves shift as a result of this exchange of ideas.

In the following case we see both the separate interpretive communities that exist within health care and how they interact and come together to reach new understandings.

Mrs. T, an 89-year-old widow in deteriorating health and with a history of recurrent strokes secondary to chronic hypertension, was hospitalized in the wake of her three children's concerns. She was progressively more disoriented, lacking in energy, and not eating well, and appeared to have recently suffered another stroke. In the hospital she was medically stabilized, but her baseline symptoms - disorientation, lethargy, and lack of appetite-continued to worsen, to the point that she only occasionally recognized her children. Over the course of the hospitalization, she also became less and less able to take nutrition by mouth. As it became clear to the medical staff (an attending physician and his team of residents) that Mrs. T would starve or die of dehydration without medical intervention 
(IV fluid and some form of artificial nutrition), they made plans to intervene as required. The nursing staff, however, expressed some concern that the patient was obviously not doing well and was progressively weakening, and that there was no reason to expect her to recover well enough to live independently, as she had for her entire life. At the same time, her family was receiving mixed messages about the care of the mother, depending upon whether the information was coming from medicine or nursing. The conflict between the doctors and nurses was sufficiently sharp that they brought in a consultation-liaison psychiatrist to help them sort out what to do. After being informed of the nurses' concerns, the psychiatrist queried the medical staff about what they actually hoped to achieve by providing nutrition and hydration to this particular patient. What became clear, as this discussion progressed, was that the doctors, after some resistance, recognized that Mrs. T was dying and that her body was, in effect, shutting down. Providing nutrition and hydration would slow down that process but could not be expected to improve her mental status or functioning in any way. The outcome of this discussion was that the doctors asked the children to come in for a meeting to discuss the mother's care. A social worker long familiar with Mrs. T-and, through her, the children-was also asked to attend. After the situation was explained to the children, they understood that their mother had led a long, rewarding life and that it was now ending. They asked for a day to sort out their feelings and, when they appeared at their mother's bedside the next day, informed the attending physician that they wanted their mother to be kept comfortable but with no further supportive interventions. The doctor informed the family that he agreed with their decision, that the social worker would be contacting them in the next few days to see how they were doing, and that the mother would be kept comfortable, as suggested. Mrs. T was moved to a hospice, where she died a week later.

Through cases such as the above, the separate fields of health care, as well as, more broadly, health care itself, evolve over time. New discoveries and technologies, problems with old ways of thinking, new theories and approaches, and changes in politics and society all encounter and challenge existing elements in one way or another. Potential new elements are then incorporated (or not) into each field-into each interpretive community - through that field's own processes for assessing, criticizing, and building upon what's known and established, to generate a new, but dynamic, status quo.

There is an important lesson for bioethics here-or, indeed, for any academic field that would try to improve or reform another. It is fair to 
say that, with its vocabulary of rights and obligations and ethical principles (or virtues or consequences, in different iterations ${ }^{3}$ ), the bioethical effort to change or illuminate ethics in health care has fallen short of expectations. Rather than building upon the existing emotional and intellectual resources of health professionals ("here's how we can improve and enrich what you already do"), the bioethics movement has attempted to displace the clinician's voice with a framework of ethical analysis and reasoning - such as one involving principles or virtues - that is fundamentally discontinuous from the language, concepts, and goals, and from the interpretive communities, of health professionals themselves. Rather than imposing new values and intellectual demands on those communities, we need to respect and build upon their strengths, including their inherent capacities for growth and change.

\section{Notes}

1. Parental anxiety about, and attention to, functional symptoms are known to trigger and reinforce the symptoms.

2. The idea here is well captured in the (2017) book by Victor Montori: Why We Revolt: A Patient Revolution for Careful and Kind Care. As the author notes in his introduction, modern health care has developed "standardized practices for patients like this, rather than caring for this patient." The controlling idea in the book is that health care systems should provide "careful and kind patient care for all." We can see this idea at work in all three of the clinical situations discussed in this chapter.

3. See comments in our concluding Chapter 11 about principlism, consequentialism, and virtue ethics.

\section{REFERENCES}

Fish, S. E. (1980). Is there a text in this class? The authority of interpretive communities. Cambridge, MA: Harvard University Press.

Fish, S. E. (1989). Doing what comes naturally: Change, rhetoric, and the practice of theory in literary and legal studies. Durham, NC: Duke University Press.

Kleinman, A. (1999). Moral experience and ethical reflection: Can ethnography reconcile them? A quandary for "the new bioethics." Daedalus, 128(4), 69-97.

Montori, V. (2017). Why we revolt: A patient revolution for careful and kind care. Rochester, MN: The Patient Revolution. 
Open Access This chapter is licensed under the terms of the Creative Commons Attribution-NonCommercial-NoDerivatives 4.0 International License (http:// creativecommons.org/licenses/by-nc-nd/4.0/), which permits any noncommercial use, sharing, distribution and reproduction in any medium or format, as long as you give appropriate credit to the original author(s) and the source, provide a link to the Creative Commons license and indicate if you modified the licensed material. You do not have permission under this license to share adapted material derived from this chapter or parts of it.

The images or other third party material in this chapter are included in the chapter's Creative Commons license, unless indicated otherwise in a credit line to the material. If material is not included in the chapter's Creative Commons license and your intended use is not permitted by statutory regulation or exceeds the permitted use, you will need to obtain permission directly from the copyright holder.

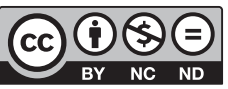

